\title{
Research for Vascular Failure: Current Topics in China
}

\author{
Ji-Guang Wang
}

\begin{abstract}
:
"Vascular failure" is a new concept, which can be used interchangeably with "vascular dysfunction". It can be evaluated with several arterial properties, such as endothelial function, arterial stiffness, wave reflection, and intima-media thickness. The current Chinese hypertension guidelines recommend some of these vascular properties for risk assessment and stratification in the management of hypertension. However, none of these measurements are routine in the clinical setting in China, because of the lack of available devices and insufficient knowledge on the clinical efficacy of these vascular measurements. In order to improve the use of these vascular measurements in clinical practice, therapeutic research is required to prove reversibility of vascular failure by either pharmacological treatments or lifestyle modifications. Other research directions include identification of emerging risk factors, risk prediction and prevention with vascular measurements, and the development of newer, more accurate and efficient devices.
\end{abstract}

\section{Key words:}

Vascular failure, Endothelial function, Arterial stiffness, Wave reflection, China

\section{Introduction}

The term "vascular failure" has been used for decades to describe "hyporeactivity to vasoconstrictors in peripheral vessels" on critical illness conditions, such as hemorrhagic or septic shock". In 2003, Schwartz SM and colleagues proposed to use "vascular failure" as a term to describe "failure of the vessel to control its mass, contractile capacity, and lumen"2). These authors hypothesized that failure to control mass and external diameter would result in hypertension or loss of lumen in atherosclerosis. In 2006, Inoue T and Node $\mathrm{K}$ proposed a new concept of "vascular failure", as a comprehensive syndrome of vascular dysfunction extending from risk factors to advanced atherosclerotic disease ${ }^{3)}$. In subsequent publications, both authors reported "vascular failure" as a pathologic mechanism for coronary artery $\mathrm{spasm}^{4)}$, with endothelial dysfunction representing the cellular mechanism $^{5}$, flow-mediated vasodilatation the diagnostic tool ${ }^{6}$, statin the treatment", and postprandial hyperglycemia the etiological factor ${ }^{8)}$ of "vascular failure". In fact, the term "vascular failure" can be used interchangeably with a longstanding term "vascular dysfunction""). The latter is described as various functional and structural abnormalities, such as endothelial dysfunction, arterial stiffness, wave reflections, and vascular hypertrophy, and can therefore be assessed by flow-mediated vasodilatation (FMD), pulse wave velocity (PWV), augmentation index, and intima-media thickness. Nonetheless, "vascular failure", like "heart failure", is probably more effective in reminding clinicians and patients that it is indeed a disease and requires careful treatment by all possible means.

Current guidelines on the management of hypertension and other cardiovascular-metabolic diseases have not yet adopted "vascular failure" as a term. Nonetheless, these guidance documents recommend several vascular measurements as target organ damage or in risk assessments. The current Chinese hypertension guidelines recommended some of the vascular measurements, such as carotid intima-media thickness and plaque, carotid femoral PWV and anklebrachial index (ABI), but not others, such as FMD or augmentation index ${ }^{10}$. The committee of the Chinese hypertension guidelines suggests that more evidence is needed for the use of vascular measurements in the management of hypertension. In this review, we will summarize several research directions for vascular abnormalities or "vascular failure" in China.

Centre for Epidemiological Studies and Clinical Trials, Shanghai Key Laboratory of Hypertension, The Shanghai Institute of Hypertension, Ruijin Hospital, Shanghai Jiaotong University School of Medicine, Shanghai, China

Corresponding author: Ji-Guang Wang, MD, PhD, jiguangwang@ aim.com

Received: February 22, 2018, Accepted: March 9, 2018

Copyright (C) 2018 Japan Society for Vascular Failure 

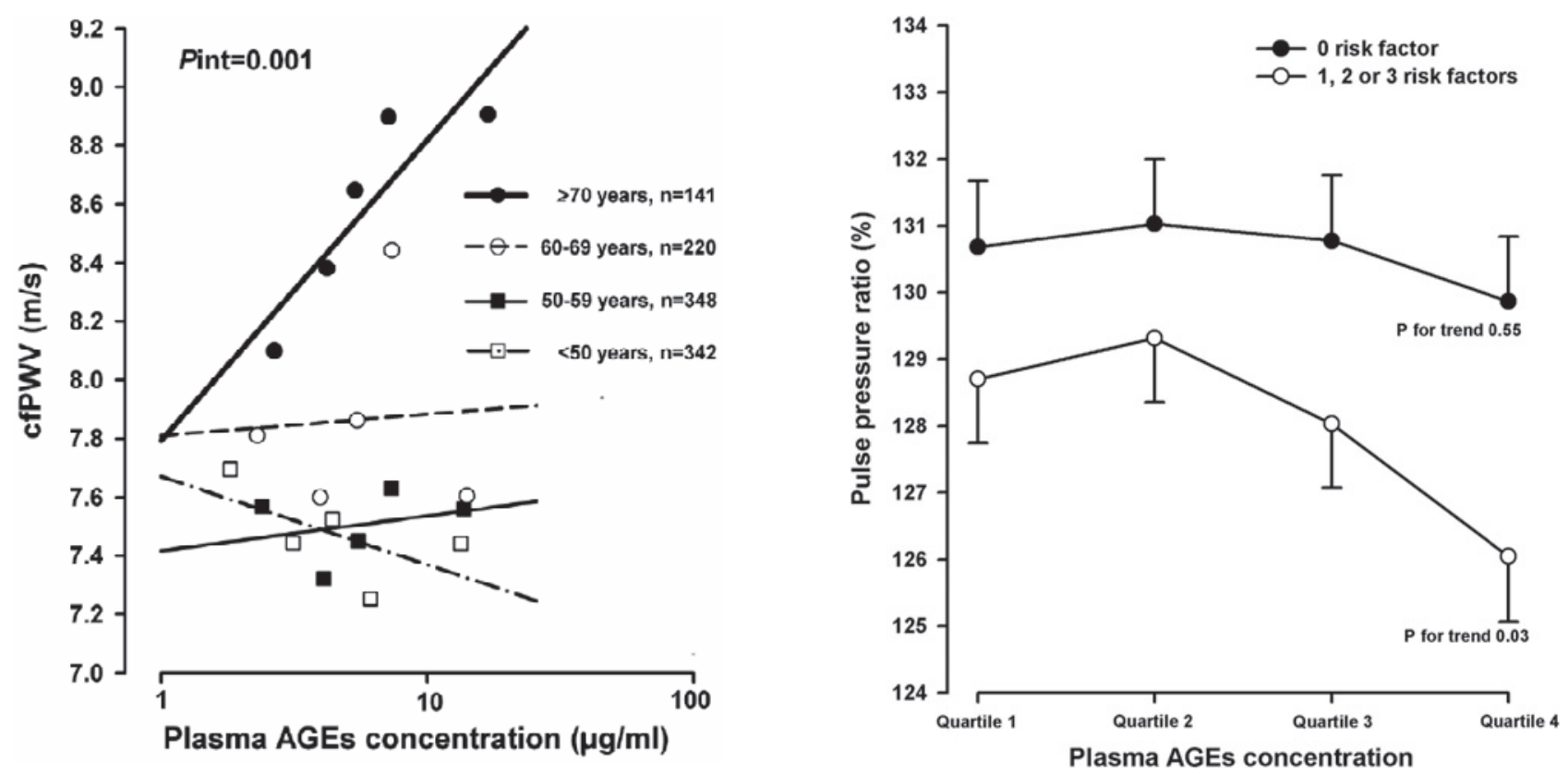

Figure 1. Relationship between carotid femoral pulse wave velocity (cfPWV) and plasma advanced glycation end products (AGEs) concentration by age subgroup (left panel) and central-to-peripheral pulse pressure ratio according to the quartile distributions of plasma AGEs concentration and the presence (circle) or absence (dot) of cardiovascular risk factors (right panel). On the left panel, the regression line was drawn according to the mean values of cfPWV per quintile of plasma AGEs concentration. The analysis was adjusted for sex, body mass index, mean arterial pressure, pulse rate, presence of diabetes mellitus or prediabetes, current smoking, use of antihypertensive drugs, and serum total-to-high density lipoprotein cholesterol ratio. Pint indicates $P$ value for the interaction between age and plasma AGEs concentration in relation to cfPWV, analyzed as continuous variables. On the right panel, values are mean, adjusted for sex, age, body height, body weight, current smoking, alcohol intake, mean arterial pressure (calculated from brachial blood pressure in the sitting position), pulse rate, plasma fasting glucose, serum total-tohigh density lipoprotein cholesterol ratio, and the use of antihypertensive drugs. Vertical lines denote standard error. $P$ values for trend are given for the participants with and without cardiovascular risk factors separately. Reproduced with permission from Huang QF, et al ${ }^{11,12)}$.

\section{Emerging risk factors}

Vascular measurements, both functional and structural, can be quantitatively and non-invasively performed, and are hence appropriate for clinical and population research. We recently investigated advanced glycation end-products (AGEs) as a cardiovascular risk factor with measurements of carotid-femoral $\mathrm{PWV}^{11)}$, augmentation index ${ }^{11)}$ and central-toperipheral pulse pressure amplification ${ }^{12)}$.

In 1051 participants of a Chinese general population, with a mean age of 55.1 years, plasma AGEs concentration was higher in 388 men than 663 women $(5.62$ vs $5.07 \mu \mathrm{g} / \mathrm{mL}, P$ $=0.02)^{11)}$. It was also higher with older age $(P \leq 0.01)$ and with higher serum total-to-high density lipoprotein cholesterol ratio $(P<0.0001)$. Overall, plasma AGEs concentration was significantly associated with central and peripheral augmentation index $(P \leq 0.02)$, but not with carotid femoral PWV $(P=0.62)$. However, there was a significant $(P=0.001)$ interaction between plasma AGEs concentration and age in relation to carotid femoral PWV (Figure 1, left panel). In participants of 70 years or older, carotid femoral PWV increased with higher levels of plasma AGEs concentration $(P$ $=0.02$ ), a result not observed in younger age groups. Taking into account the results of our study ${ }^{11)}$ and other studies ${ }^{13,14)}$, we believe that AGEs accumulate with aging and high cholesterol, and are associated with arterial wave reflections and, in an age-dependent manner, arterial stiffness.

In further analysis in the same study population, plasma AGEs concentration was associated with central-to-brachial blood pressure amplification, as expressed by central-tobrachial pulse pressure ratio ${ }^{12}$. Each 10-time increase in plasma AGEs concentration was associated with $2.39 \%$ lower central-to-brachial pulse pressure ratio $(P=0.03)$. This association was more prominent in the presence of several common cardiovascular risk factors, such as overweight and obesity $(-4.89 \%, \quad P=0.009)$, diabetes and prediabetes $(-6.26 \%, P=0.10)$ and current smoking $(-6.68 \%, P=0.045$,

Figure 1, right panel).

Our study results should encourage further research on the risk and reversibility of AGEs accumulation in cardiovascular prevention. In spite of the failure to bring the crosslink breaker, alagebrium, to a successful pharmaceutical product, the pathway of AGEs should not be dismissed in the development of novel drugs for vascular protection and prevention $^{15}$. 

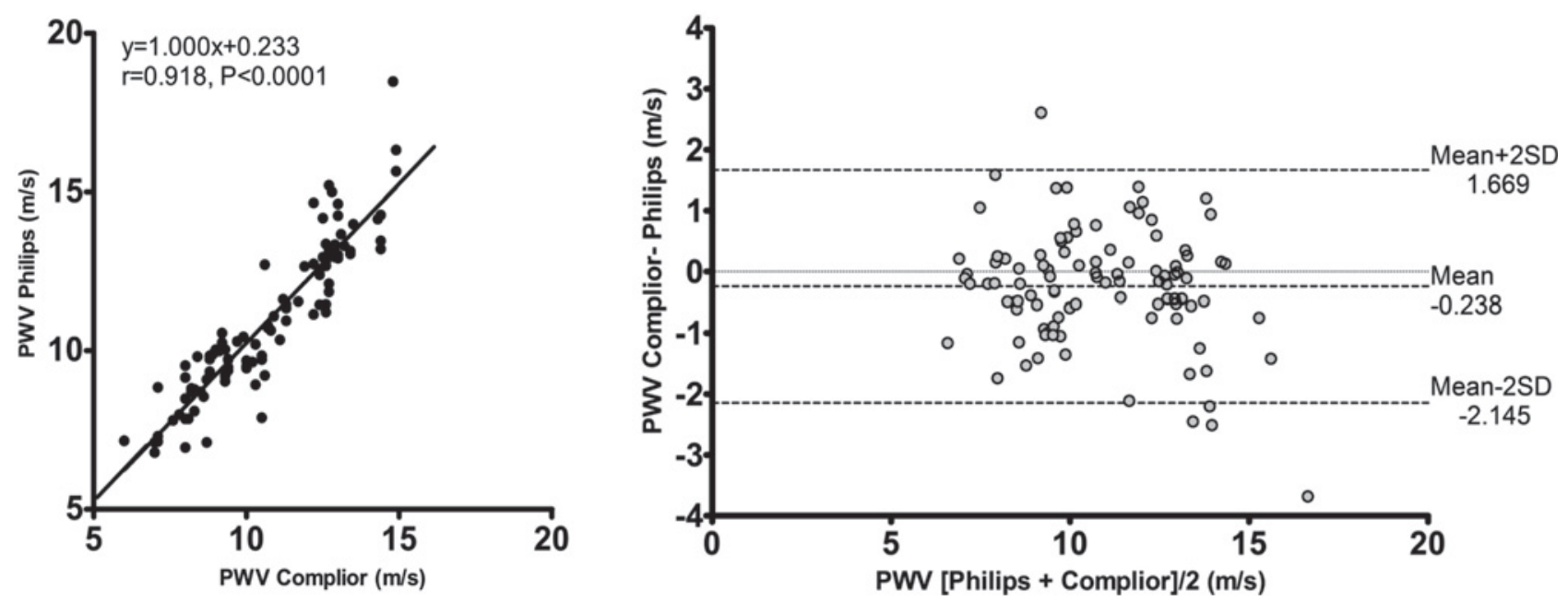

Figure 2. Correlation between pulse wave velocity $(P W V, m / s)$ measured with the Philips prototype and Complior device (left panel) and Bland-Altman analysis of the difference between pulse wave velocity (PWV) values obtained by the two devices against the average of the two PWV values (right panel). On the left panel, the regression line was drawn and the regression equation is given with correlation coefficient and $P$ value. On the right panel, the lines of the mean difference and the mean \pm 2 standard deviations (SD) were drawn. Reproduced with permission from Xu SK, et al ${ }^{25)}$.

\section{Risk stratification and prediction}

There is strong evidence that vascular measurements can be used for cardiovascular risk stratification and prediction. In our elderly ( $\geq 60$ years) population cohort, we studied prognostic significance of several vascular measurements.

In 3876 elderly Chinese participants, we found that brachial ankle PWV (mean $\pm \mathrm{SD}, 17.8 \pm 4.0 \mathrm{~m} / \mathrm{s}$ ) was significantly $(\mathrm{P}<0.0001)$ associated with all-cause $(\mathrm{n}=316)$, cardiovascular $(n=148)$, stroke $(n=46)$ and non-cardiovascular mortality $(n=168)$ during a median follow-up of 5.9 years $^{16)}$. In further adjusted analysis, the participants in the top decile of brachial ankle PWV $(23.3-39.3 \mathrm{~m} / \mathrm{s})$ had a significantly $(P \leq$ 0.003 ) higher risk of all-cause mortality (hazard ratio relative to the whole study population $1.56,95 \%$ confidence interval 1.16-2.08), especially in hypertensive patients (hazard ratio $1.86,95 \%$ confidence interval $1.31-2.64, P=0.02$ for the interaction between brachial ankle PWV and hypertension). Similar trends were observed for cardiovascular, stroke and non-cardiovascular mortality, though statistical significance was not reached $(P \geq 0.08)$.

In our elderly population cohort, we also studied the elongated upstroke time in pulse waves in the lower extremities for the diagnosis of peripheral arterial disease and for the prediction of total and cardiovascular mortality ${ }^{17}$. Upstroke time per cardiac cycle at baseline (mean \pm SD, $16.4 \% \pm$ $3.1 \%)$ was significantly $(\mathrm{P}<0.0001)$ associated with $\mathrm{ABI}$ in men $(n=1803, r=-0.44)$ and women $(n=2252, r=-0.32)$. During the 5.9 years (median) of follow-up, all-cause and cardiovascular deaths occurred in 366 and 183 participants, respectively. In adjusted Cox regression analyses, an upstroke time per cardiac cycle of $\geq 21.7 \% \quad(n=219,5.4 \%)$ significantly $(\mathrm{P}<0.0001)$ predicted total and cardiovascular mortality. The corresponding hazard ratios were 1.98 (95\% confidence interval, 1.48-2.65) and 2.29 (1.58-3.32), respectively, as compared with that of $2.10(1.48-3.00)$ and 2.44 (1.57$3.79)$, respectively, associated with an $\mathrm{ABI} \leq 0.90 \quad(\mathrm{n}=115$, $2.8 \%)$.

The results of these studies corroborated the clinical usefulness of brachial ankle PWV in the evaluation of cardiovascular risk and prediction of cardiovascular events, even in the elderly. In addition, the upstroke time per cardiac cycle in pulse waves may provide incremental predictive value over and above some of the conventional measurements, such as PWV and ABI.

\section{Therapeutic treatment}

Risk stratification and prediction is important. However, a clinically useful measurement should also be able to define clinical abnormalities and guide treatment. At present, few vascular measurements have fully met these criteria. Nonetheless, several studies have demonstrated that central blood pressure $^{18)}$ and $\mathrm{FMD}^{19-21)}$ might be useful in the therapeutic management of hypertension and cardiovascular disease.

There is some evidence that $\beta$-blockers are less effective than other classes of antihypertensive drugs in central blood pressure lowering ${ }^{18}$. We performed a meta-analysis of trials that compared $\beta$-blockers with other classes of antihypertensive drugs in order to quantify to what extent the heart rate slowing effect of $\beta$-blockers accounts for the reduction of central augmentation index and central blood pressure and, in turn, the risk of stroke ${ }^{22}$. In 754 patients across 9 trials, $\beta$-blockers were less effective than other classes of antihypertensive drugs in reducing central systolic blood pressure (3.75 mm Hg, $P=0.03$ ) and central augmentation index $(8.57 \%, P<0.001)$. In a meta-regression analysis of these 9 trials, the baseline-adjusted difference in heart rate between randomized groups was significantly associated with central augmentation index $(5.00 \%$ increase for each 10 beats per 
minute decrease in heart rate, $P=0.02$ ), which was significantly associated with central systolic blood pressure (1.16 $\mathrm{mm} \mathrm{Hg}$ increase for each $1 \%$ increase in central augmentation index, $P=0.009)$. In a meta-analysis of 5 outcome trials, the pooled odds ratio of stroke was 1.26 (95\% CI 1.14 to $1.38, P<0.001)$ for $\beta$-blockers versus other classes of antihypertensive drugs. This risk difference could be accounted for by the difference in central systolic blood pressure derived from the meta-regression analysis, but not by the observed difference in brachial systolic blood pressure.

FMD has been used in the research setting since early $1990 \mathrm{~s}^{23}$. However, it has not yet been widely used in the clinical setting. We recently utilized this technique to measure endothelial function and to guide treatment in a 13-year old boy with a 3-year history of difficult-to-control isolated systolic hypertension (unpublished data). After exclusion of known secondary causes of hypertension, such as aortic coarctation, renovascular and renal parenchymal hypertension, and abnormalities in the adrenal glands, we measured central blood pressure and FMD. Central blood pressure was similarly elevated as the brachial blood pressure, indicating true systemic hypertension. FMD was close to $0.0 \%$, meaning that brachial diameter changed negligibly from systole to diastole. The patient's father was a heavy smoker. A previous study in adolescents clearly demonstrated that passive smoking was an important risk factor for FMD decline ${ }^{24)}$. We therefore strongly advised a non-smoking lifestyle, and prescribed a high dose of vasoactive drugs, i.e. an angiotensin-converting enzyme inhibitor and a dihydropyridine calcium channel blocker. With the lifestyle change and drug treatment, the boy's FMD gradually increased to $4 \%$ and $7 \%$, after 2 and 4 years of treatment, respectively. His blood pressure could be well controlled with a low dose of antihypertensive drugs.

A clinical implication of these study results is that we can and should consider these vascular measurements in guiding antihypertensive therapy or other cardiovascular therapeutic treatments.

\section{Newer devices}

One of the major advances in the field of "vascular failure" is the development of several imaging and non-imaging devices for the measurement of vascular structure and function. Nonetheless, more devices are still needed to improve accuracy and/or efficiency of measurement.

We recently conducted a comparative study to validate the Philips prototype for carotid femoral PWV measurement against the well-accepted and widely-used Complior device (Alam Medical, Paris, France) ${ }^{25}$. The Philips prototype is equipped with probes that consist of multiple piezo-electric sensors. The probe chooses the best signal detected by one of the multiple sensors. This approach may potentially improve accuracy of measurements made, especially in difficult cases. We recruited 33 participants with a wide distribution of PWV, and obtained 99 paired PWV values. The correla- tion coefficient for PWV measured with the two devices was $0.92(P<0.0001$, Figure 2, left panel). Compared with the Complior device, the Philips prototype slightly overestimated PWV by 0.24 ( \pm 2 standard deviations, \pm 1.91$) \mathrm{m} / \mathrm{s}$, especially when PWV was high (Figure 2, right panel).

Current devices for PWV measurement work with the piezoelectric pressure mechano-transducing technology, the Millar tonometry for carotid femoral PWV or the oscillometry for brachial ankle PWV. New devices are being developed with an optical non-contact probe for the detection of arterial distension waveform ${ }^{26}$. This new technology does not only improve accuracy in signal acquisition, but will likely also make it possible to measure some PWV measurements in the sitting position. The latter advantage may substantially simplify the procedure and improve the efficiency of PWV measurement.

\section{Conclusions and perspectives}

"Vascular failure" is a fascinating new area of research. This new term will likely help move this area from technical diagnostics to clinical management. However, a lot of work still has to be done and newer technology and devices are always required. Clinical outcome studies are essential for the use of vascular measurements in cardiovascular prediction and prevention, and for making "vascular failure" a clinical entity with specific management.

\section{Sources of Funding}

The present study was financially supported by grants from the National Natural Science Foundation of China (91639203), the Ministry of Science and Technology (2013 CB530701), Beijing China, the Shanghai Commission of Science and Technology (15XD1503200), and the Shanghai Bureau of Health (XBR2011004), Shanghai, China.

\section{Conflicts of Interest}

The author declares no conflict of interest.

\section{References}

1. Davidson CS, Lewis JH, Tagnon HJ, Adams MA, Taylor FHL. Medical shock: Abnormal biochemical changes in patients with severe, acute medical illnesses, with and without peripheral vascular failure. N Engl J Med 1946; 234: 279-88.

2. Schwartz SM, Geary RL, Adams LD. Vascular failure: a hypothesis. Curr Atheroscler Rep 2003; 5: 201-7.

3. Inoue T, Node K. Vascular failure: A new clinical entity for vascular disease. J Hypertens 2006; 24: 2121-30.

4. Kawano H, Node K. The role of vascular failure in coronary artery spasm. J Cardiol 2011; 57: 2-7.

5. Hirase T, Node K. Endothelial dysfunction as a cellular mechanism for vascular failure. Am J Physiol Heart Circ Physiol 2012; 302: H499-505.

6. Inoue T, Matsuoka H, Higashi Y, Ueda S, Sata M, Shimada KE, et al; Vascular Failure Workshop Group. Flow-mediated vasodilation as a diagnostic modality for vascular failure. Hypertens Res 2008; 31: 2105-13.

7. Inoue T, Node K. Statin therapy for vascular failure. Cardiovasc 
Drugs Ther 2007; 21: 281-95.

8. Node K, Inoue T. Postprandial hyperglycemia as an etiological factor in vascular failure. Cardiovasc Diabetol 2009; 8: 23.

9. Grand'Maison S, Pilote L, Okano M, Landry T, Dayan N. Markers of vascular dysfunction after hypertensive disorders of pregnancy: a systematic review and meta-analysis. Hypertension 2016; 68: 1447-58.

10. Liu LS; Writing Group of 2010 Chinese Guidelines for the Management of Hypertension. 2010 Chinese guidelines for the management of hypertension. Chin J Cardiol 2011; 39: 579-615(Chinese).

11. Huang QF, Sheng CS, Liu M, Li FH, Li Y, Wang JG. Arterial stiffness and wave reflections in relation to plasma advanced glycation end products in a Chinese population. Am J Hypertens 2013; 26: 754-61.

12. Huang QF, Sheng CS, Kang YY, Zhang L, Wang S, Li FK, et al. Central and peripheral blood pressures in relation to plasma advanced glycation end products in a Chinese population. J Hum Hypertens 2016; 30: 430-5.

13. McNulty M, Mahmud A, Feely J. Advanced glycation endproducts and arterial stiffness in hypertension. Am J Hypertens 2007; 20: 242-7.

14. Semba RD, Najjar SS, Sun K, Lakatta EG, Ferrucci L. Serum carboxymethyl-lysine, an advanced glycation end product, is associated with increased aortic pulse wave velocity in adults. Am J Hypertens 2009; 22: 74-9.

15. Zieman SJ, Melenovsky V, Clattenburg L, Corretti MC, Capriotti A, Gerstenblith G, et al. Advanced glycation endproduct crosslink breaker (alagebrium) improves endothelial function in patients with isolated systolic hypertension. J Hypertens 2007; 25: 577-83.

16. Sheng CS, Li Y, Li LH, Huang QF, Zeng WF, Kang YY, et al. Brachial-ankle pulse wave velocity as a predictor of mortality in elderly Chinese. Hypertension 2014; 64: 1124-30.

17. Sheng CS, Li Y, Huang QF, Kang YY, Li FK, Wang JG. Pulse waves in the lower extremities as a diagnostic tool of peripheral arterial disease and predictor of mortality in elderly Chinese. Hy- pertension 2016; 67: 527-34.

18. Williams B, Lacy PS, Cruickshank JK, Collier D, Hughes AD, Stanton A, et al; CAFE and ASCOT Investigators. Impact of statin therapy on central aortic pressures and hemodynamics: principal results of the Conduit Artery Function Evaluation-Lipid-Lowering Arm (CAFE-LLA) Study. Circulation 2009; 119: 53-61.

19. Ding FH, Li Y, Li LH, Wang JG. Impact of heart rate on central hemodynamics and stroke: a meta-analysis of $\beta$-blocker trials. Am J Hypertens 2013; 26: 118-25.

20. Muiesan ML, Salvetti M, Monteduro C, Rizzoni D, Zulli R, Corbellini $\mathrm{C}$, et al. Effect of treatment on flow-dependent vasodilation of the brachial artery in essential hypertension. Hypertension 1999; 33: 575-80.

21. Nissen SE, Tuzcu EM, Schoenhagen P, Brown BG, Ganz P, Vogel RA, et al; REVERSAL Investigators. Effect of intensive compared with moderate lipid-lowering therapy on progression of coronary atherosclerosis: a randomized controlled trial. JAMA 2004; 291: 1071-80.

22. Moriguchi J, Itoh H, Harada S, Takeda K, Hatta T, Nakata T, et al. Low frequency regular exercise improves flow-mediated dilatation of subjects with mild hypertension. Hypertens Res 2005; 28: 31521.

23. Celermajer DS, Sorensen KE, Gooch VM, Spiegelhalter DJ, Miller OI, Sullivan ID, et al. Non-invasive detection of endothelial dysfunction in children and adults at risk of atherosclerosis. Lancet 1992; 340: 1111-5.

24. Celermajer DS, Adams MR, Clarkson P, Robinson J, McCredie R, Donald A, et al. Passive smoking and impaired endotheliumdependent arterial dilatation in healthy young adults. $\mathrm{N}$ Engl $\mathrm{J}$ Med 1996; 334: 150-4.

25. Xu SK, Cheng YB, Liu CY, Li Y, Wang JG. Validation of a piezoelectric sensor array-based device for measurement of carotidfemoral pulse wave velocity: the Philips Prototype. Pulse 2017; 5: 161-8.

26. Pereira T, Correia C, Cardoso J. Novel methods for pulse wave velocity measurement. J Med Biol Eng 2015; 35: 555-65. 